\title{
A norbornyl route to some novel seven-membered iminocyclitols
}

\author{
Goverdhan Mehta* and Sripada Lakshminath \\ Department of Organic Chemistry, Indian Institute of Science, Bangalore 560 012, India
}

\begin{abstract}
New hydroxy azepanes with an additional hydroxymethyl side arm have been conceived and their syntheses achieved from a suitably functionalized cyclohexanoid building-block extracted from the norbornyl framework. The new iminocyclitols (homoisofagomine derivatives) exhibit weak but selective inhibitory activity in enzyme assays.
\end{abstract}

Replacement of ring oxygen with basic nitrogen in sugars gives rise to azasugars (also referred to as imino sugars) and these entities have not only been found in Nature but also aroused a great deal of synthetic interest in recent years because of their ability to inhibit glycosidases. ${ }^{1}$ While deoxynojirimycin 1 constitutes a well known example of a potent naturally occuring glycosidase inhibitor, 1-deoxyfuconojirimycin $\mathbf{2}$ is a synthetic analogue ${ }^{2 \mathrm{a}}$ and the most powerful inhibitor of $\alpha$-fucosidases known to date. This impressive biological activity profile of iminosugars and their potential for therapeutic applications has stimulated interest in the synthesis of newer analogues through manipulation of the number and stereochemical disposition of the hydroxyl groups, relocation of the hydroxymethyl arm as in the potent inhibitor isofagomine $3,{ }^{2 \mathrm{~b}, \mathrm{c}, \mathrm{e}}$ introduction of additional ring nitrogen atom as in the diazasugar $4^{2 \mathrm{~d}}$ among others. More recently, iminocyclitols based on seven-membered rings having the same carbon content as the azasugars but endowed with the conformational advantage of a more flexible azepane ring that could lead to favorable binding to the active site of the enzyme have been introduced. ${ }^{3}$ Indeed, iminocyclitols 5-7 have shown promising glycosidase inhibition profiles. We have visualized another variant of the seven-membered iminocyclitols with an additional binding site in the form of a hydroxymethyl arm, which can be regarded as a hybrid of the polyhydroxyazepanes and isofagomine 3. Syntheses of several of these new iminocyclitols $\mathbf{8}$ form the subject matter of this letter.
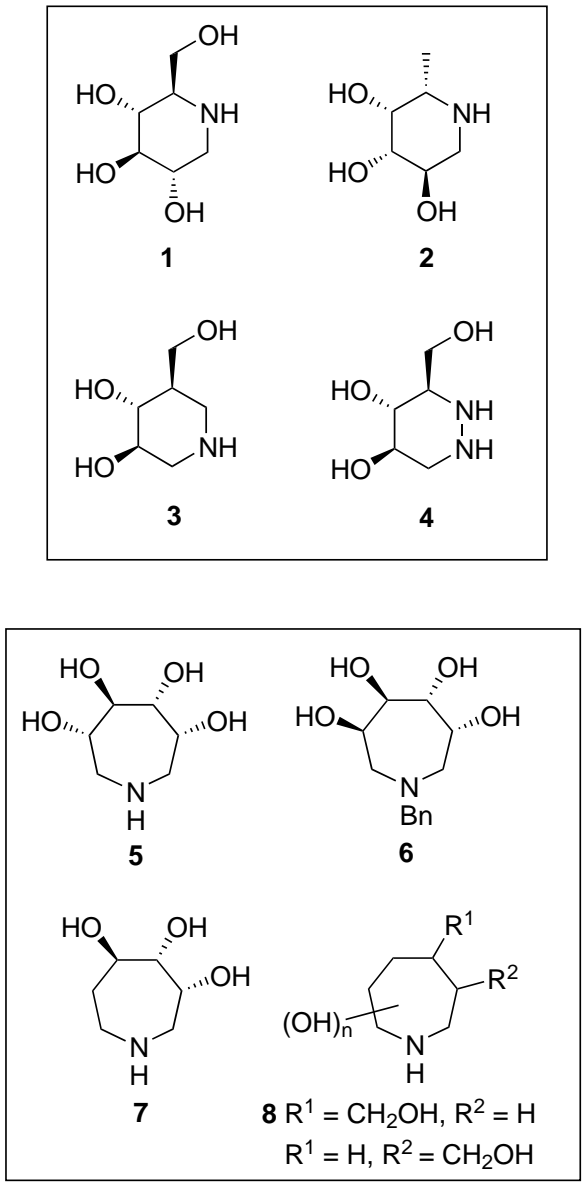

Recently, we have delineated a convenient access to a functionalized cyclohexenoid $\mathbf{1 0}$ from a norbornyl precursor 9 involving a fragmentation protocol. ${ }^{4 a}$ It has been shown that $\mathbf{1 0}$ is a versatile building block, comparable to the cyclohexadiene-diols derived through

* Corresponding author. Fax: +91-80-3600936; e-mail: gm@ orgchem.iisc.ernet.in 
microbial hydroxylation of arenes, ${ }^{5}$ for the synthesis of diverse cyclitols and related structures. ${ }^{4}$ Herein, we further demonstrate the utility of $\mathbf{1 0}$ in the synthesis of novel hydroxyazepanes related to $\mathbf{8}$ (Scheme 1). Elaboration of $\mathbf{1 0}$ to azepanes required oxidative cleavage of the cyclohexanoid ring to set-up either a double reductive amination or inter- and intramolecular $N$-alkylations to deliver system $\mathbf{8}$. Towards this end, 10 was transformed to the cis-1,2-diol $\mathbf{1 1}^{6}$ via $\mathrm{LiAlH}_{4}$ reduction, protection of the resulting primary hydroxyl functionality and stereoselective catalytic $\mathrm{OsO}_{4}$ dihydroxylation. Periodate mediated oxidative cleavage to the dialdehyde, sodium borohydride reduction and mesylation on $\mathbf{1 1}$ proceeded uneventfully to furnish the dimesylate 12 (Scheme 1). ${ }^{6}$ Exposure of $\mathbf{1 2}$ to $p$-toluenesulfonamide under phase transfer conditions resulted in smooth inter- and the intramolecular $N$-alkylations to 13. The $N$-tosyl group in $\mathbf{1 3}$ could be reductively removed with sodium naphthalenide and the resulting free amine was characterized as the acetamide 14, which existed as a mixture of two rotamers. Deprotection maneuvers in $\mathbf{1 4}$ led to a homoisofagomine (carbon inserted between nitrogen and hydroxymethyl group of 3 ) derivative 15 (Scheme 1). ${ }^{6}$ An isomeric homoisofagomine derivative with a carbon inserted between the hydroxymethyl group and the neighboring hydroxy group was also prepared from 10 through a tactically altered route. Cyclohexenoid $\mathbf{1 0}$ was first elaborated to $\mathbf{1 6}$ through $\mathrm{LiAlH}_{4}$ reduction, stereoselective catalytic dihydroxylation and acetylation (Scheme 2). ${ }^{6}$ Acetonide deprotection in $\mathbf{1 6}$ gave $\mathbf{1 7}$ and the 1,2-diol functionality was subjected to periodate cleavage to
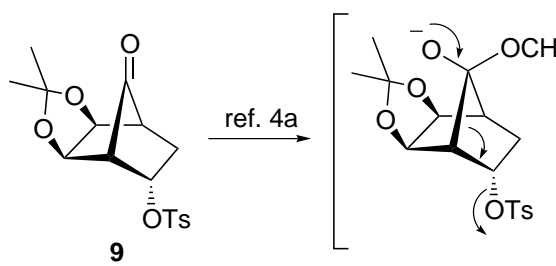

ÖTs

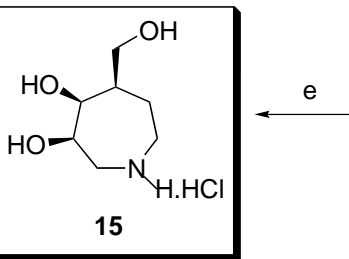<smiles>CC1(C)OC2CN(C(C)(C)C)CCC(CO)C2O1</smiles>

14<smiles>CC(=O)C1CC=CC2OC(C)(C)OC21</smiles>

10<smiles>[TeH][TeH]</smiles>
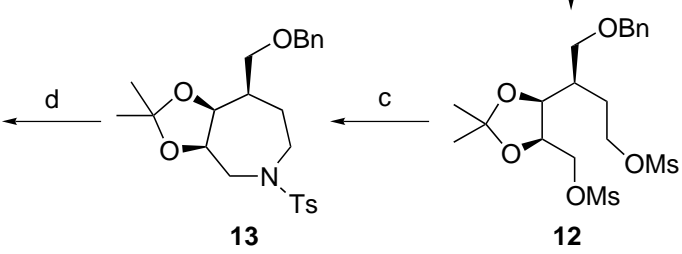

Scheme 1. Reagents and conditions: (a) i. LAH, THF, $0-5^{\circ} \mathrm{C}, 1 \mathrm{~h}, 90 \%$, ii. $\mathrm{BnBr}, \mathrm{NaH}, \mathrm{THF}, \mathrm{rt}, 6 \mathrm{~h}, 95 \%$, iii. OsO $4, \mathrm{NMMO}$, $\mathrm{Me}_{2} \mathrm{CO}-\mathrm{H}_{2} \mathrm{O}$ (4:1), rt, overnight, 83\%; (b) i. $\mathrm{NaIO}_{4}$-silica gel, DCM, $0^{\circ} \mathrm{C}, 2 \mathrm{~h}$, ii. $\mathrm{NaBH}_{4}, \mathrm{MeOH}^{\circ} 0^{\circ}, 1 \mathrm{~h}$, iii. $\mathrm{MsCl}, \mathrm{Et}{ }_{3} \mathrm{~N}$, DCM, $0^{\circ} \mathrm{C}, 1.5 \mathrm{~h}, 55 \%$ (three steps); (c) $p \mathrm{TsNH}_{2}, \mathrm{KOH}, \mathrm{TBAI}, \mathrm{C}_{6} \mathrm{H}_{6}-\mathrm{H}_{2} \mathrm{O}$ (9:1), reflux, $24 \mathrm{~h}, 60 \%$; (d) i. Na-naphthalenide, DME, $-60^{\circ} \mathrm{C}, 1 \mathrm{~h}$, ii. $\mathrm{Ac}_{2} \mathrm{O}, \mathrm{Py}, \mathrm{rt}$, overnight, 67\% (two steps); (e) i. $\mathrm{H}_{2}, \mathrm{Pd} / \mathrm{C}, \mathrm{EtOH}, 12 \mathrm{~h}$, ii. $1 \mathrm{~N} \mathrm{HCl}, 90^{\circ} \mathrm{C}, 85 \%$ (two steps).

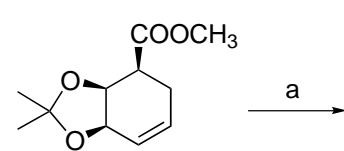

10

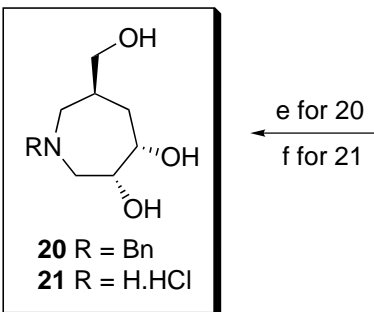<smiles>CC(=O)OCC1C[C@H](OC(C)=O)[C@H](OC(C)=O)[C@@H]2OC(C)(C)O[C@H]12</smiles>

16<smiles>CC(=O)OCC1CN(Cc2ccccc2)C[C@H](OC(C)=O)[C@@H]1OC(C)=O</smiles><smiles>C1CCCCC1</smiles><smiles>CC(=O)OCC1C[C@H](OC(C)=O)[C@H](O)[C@H](O)[C@H]1O</smiles>

17

c<smiles>CC(=O)OC[C@H](C)C[C@@H](OC(C)=O)[C@@H](C)O</smiles>

Scheme 2. Reagents and conditions: (a) i. LAH, THF, $0-5^{\circ} \mathrm{C}, 1 \mathrm{~h}, 90 \%$, ii. $\mathrm{OsO}_{4}, \mathrm{NMMO}_{2} \mathrm{Me}_{2} \mathrm{CO}-\mathrm{H}_{2} \mathrm{O}(4: 1)$, rt, overnight, $82 \%$, iii. $\mathrm{Ac}_{2} \mathrm{O}$, DMAP, DCM, rt, 10 h, 78\%; (b) Amberlyst-15, THF- $\mathrm{H}_{2} \mathrm{O}$ (2:3), rt, 12 h, 85\%; (c) $\mathrm{NaIO}_{4}-$ silica gel, DCM, $0{ }^{\circ} \mathrm{C}, 2$ h; (d) $\mathrm{BnNH}_{2}, \mathrm{AcOH}, \mathrm{NaCNBH}_{3}, \mathrm{MeOH}, 12 \mathrm{~h}, 35 \%$ (two steps); (e) $\mathrm{K}_{2} \mathrm{CO}_{3}, \mathrm{MeOH}, \mathrm{rt}, 6 \mathrm{~h}, 95 \%$; (f) i. H $, \mathrm{Pd} / \mathrm{C}, \mathrm{EtOH}, \mathrm{rt}, 5$ h, ii. $1 \mathrm{~N} \mathrm{HCl}, 90^{\circ} \mathrm{C}, 24 \mathrm{~h}, 90 \%$. 


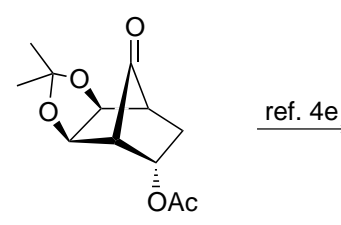

22

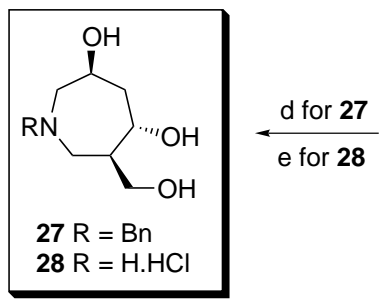

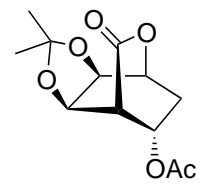

23

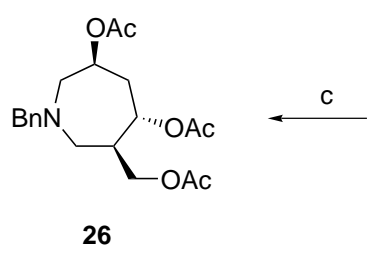<smiles>CC(=O)OCC1CC(OC(C)=O)C(O)C(O)C1OC(C)=O</smiles>

24

b<smiles>CC(=O)OC[C@@H](C=O)[C@@H](C[C@@H](C=O)OC(C)=O)OC(C)=O</smiles>

25

Scheme 3. Reagents and conditions: (a) i. LAH, THF, $0-5^{\circ} \mathrm{C}, 3 \mathrm{~h}, 70 \%$, ii. $\mathrm{Ac}_{2} \mathrm{O}, \mathrm{DMAP}, \mathrm{DCM}, \mathrm{rt}, 12 \mathrm{~h}, 89 \%$, iii. Amberlyst- 15 , THF- $\mathrm{H}_{2} \mathrm{O}$ (2:3), rt, 12 h, 85\%; (b) $\mathrm{NaIO}_{4}$-silica gel, DCM, $0^{\circ} \mathrm{C}, 2$ h; (c) $\mathrm{BnNH}_{2}, \mathrm{AcOH}, \mathrm{NaCNBH}_{3}, \mathrm{MeOH}, 15 \mathrm{~h}, 30 \%$ (two steps); (d) $\mathrm{K}_{2} \mathrm{CO}_{3}, \mathrm{MeOH}, \mathrm{rt}, 6 \mathrm{~h}$, quant.; (e) i. $\mathrm{H}_{2}, \mathrm{Pd} / \mathrm{C}, \mathrm{EtOH}, \mathrm{rt}, 5 \mathrm{~h}$, ii. $1 \mathrm{~N} \mathrm{HCl,} 90^{\circ} \mathrm{C}, 20$ h, $90 \%$.

deliver the intermediate dialdehyde 18, which was directly subjected to a double-reductive amination ${ }^{7}$ to give the azepane $\mathbf{1 9}$ as the major product (Scheme 2). ${ }^{6}$ While acetate hydrolysis in $\mathbf{1 9}$ gave the $N$-benzyl-protected trihydroxy azepane $\mathbf{2 0}$, reductive removal of the $N$-benzyl group and deprotection gave the trihydroxy azepane 21 (Scheme 2). ${ }^{6}$

Another homoisofagomine related to $\mathbf{2 1}$ with a 'skipped hydroxyl' pattern was accessed from the endo-norbornyl derivative 22. Regioselective Baeyer-Villiger oxidation of $\mathbf{2 2}$ gave $\mathbf{2 3}$ as the major product $(87: 13$ mixture of regioisomers). A sequence consisting of $\mathrm{LiAlH}_{4}$ reduction, per-acetylation and acetonide deprotection in 23 led to cyclohexane-1,2-diol 24 (Scheme 3). ${ }^{6}$ Periodate cleavage in $\mathbf{2 4}$ led to an intermediate dialdehyde 25, which was directly subjected to double-reductive amination with benzylamine to give the azepane triacetate $\mathbf{2 6}$ as the major product. Acetate hydrolysis in 26 gave the $N$-benzyl trihydroxy derivative 27. On the other hand, reductive removal of the $N$-benzyl group and acetate deprotection furnished the trihydroxyazepane 28 (Scheme 3). ${ }^{6}$

The new homoisofagomine analogues 15, 20, 21, 27 and 28 were assayed for glycosidase inhibition against a set of six commonly used enzymes ( $\alpha$ - and $\beta$-glucosidase, $\alpha$ - and $\beta$-galactosidase, and $\alpha$ - and $\beta$-mannosidase) following standard protocols. All the substrates examined exhibited weak but selective inhibition with 20 and 21 showing relatively better activity against $\beta$-glucosidase $\left(K_{\mathrm{i}}=470 \mu \mathrm{M}\right)$ and $\alpha$-galactosidase $\left(K_{\mathrm{i}}=600 \mu \mathrm{M}\right)$, respectively. It is quite interesting that while the $N$-benzyl derivative 20 shows inhibition of $\beta$-glucosidase, the unprotected $\mathbf{2 1}$ inhibits $\alpha$-galactosidase. This change in enzyme selectivity in the case of $\mathbf{2 0}$ and $\mathbf{2 1}$ mediated only by the protective group, without any alteration in the disposition or stereochemistry of the hydroxyl groups, is quite unusual and may have useful implications. We have observed similar selectivity in the case of
27 and 28, as well with the former exhibiting weak inhibition of $\beta$-glucosidase and the latter inhibiting $\alpha$-glucosidase. ${ }^{8}$

In conclusion, we have amplified the synthetic utility of the cyclohexenoid building block $\mathbf{1 0}$ by devising stereoselective routes to new hydroxyazepanes with a homoisofagomine framework. While the azepanes reported here have only weak glycosidase inhibitory activity compared to those reported earlier, our results of enzymatic assays reveal the importance of $N$-substitution in modulating selectivity and inhibition efficacy.

\section{Acknowledgements}

We thank the Chemical Biology Unit of the JNCASR for the support of this research. One of us (S.L.) thanks CSIR for the award of research fellowship.

\section{References}

1. Recent reviews: (a) Hughes, A. B.; Rudge, A. J. Nat. Prod. Rep. 1994, 135; (b) Jacob, G. S. Curr. Opin. Struct. Biol. 1995, 605; (c) Ganem, B. Acc. Chem. Res. 1996, 29, 340; (d) Bols, M. Acc. Chem. Res. 1998, 31, 1; (e) Heightman, T. D.; Vasella, A. T. Angew. Chem., Int. Ed. Engl. 1999, 38, 750; (f) Sears, P.; Wong, C.-H. Angew. Chem., Int. Ed. Engl. 1999, 38, 2300.

2. (a) Fleet, G. W.; Namguong, S. K.; Barker, C.; Bainses, S.; Jacob, G. S.; Winchester, B. Tetrahedron Lett. 1989, 30, 4439; (b) Ichikawa, Y.; Igarashi, Y.; Ichikawa, M.; Suhara, Y. J. Am. Chem. Soc. 1998, 120, 3007; (c) Jespersen, T. M.; Dong, W.; Sierks, M. R.; Skrydsurp, T.; Lundt, I.; Bols, M. Angew. Chem., Int. Ed. Engl. 1994, 33, 1778; (d) Ernholt, B. V.; Thomson, I. B.; Jensen, K.; Bols, M. Synlett 1999, 6, 701; (e) Hensen, S. U.; Bols, M. J. Chem. Soc., Perkin Trans. 1 2000, 911. 
3. (a) Paulsen, H.; Todt, K. Chem. Ber. 1967, 100, 512; (b) Farr, R. A.; Holland, A. K.; Hubor, E. W.; Peet, N. P.; Weintraub, P. M. Tetrahedron 1994, 50, 1044; (c) Poitout, L.; Le Merrer, Y.; Depezay, J.-C. Tetrahedron Lett. 1994, 35, 3293; (d) Lohray, B. B.; Jayamma, Y.; Chatterji, M. J. Org. Chem. 1995, 60, 5958; (e) Moris-Varas, F.; Qian, X.-H.; Wong, C.-H. J. Am. Chem. Soc. 1996, 118, 7467; (f) Qian, X.-H.; Moris-Varas, F.; Wong, C.-H. Bioorg. Med. Chem. Lett. 1996, 6, 1667; (g) Fuentes, J.; Olano, D.; Pradera, M. A. Tetrahedron Lett. 1999, 40, 4063; (h) Gauzy, L.; Le Merrer, Y.; Depezay, J.-C.; Clere, F.; Mignani, S. Tetrahedron Lett. 1999, 40, 6005; (i) Anderson, S. M.; Ekhart, C.; Lundt, I.; Stutz, A. E. Carbohydr. Res. 2000, 326, 22; (j) Painter, G. F.; Falshaw, A. J. Chem. Soc., Perkin Trans. 1 2000, 1157; (k) Tezuka, K.; Compain, P.; Martin, O. R. Synlett 2000, 1837; (l) Gallos, J. K.; Demeroudi, S. C.; Stathopoulou, C. C.; Dellios, C. C. Tetrahedron Lett. 2001, 42, 7497.

4. (a) Mehta, G.; Mohal, N.; Lakshminath, S. Tetrahedron Lett. 2000, 41, 3505; (b) Mehta, G.; Lakshminath, S. Tetrahedron Lett. 2000, 41, 3509; (c) Mehta, G.; Mohal, N. Tetrahedron Lett. 2000, 41, 5741; (d) Mehta, G.; Mohal, N. Tetrahedron Lett. 2000, 41, 5747; (e) Mehta, G.; Talukdar, P.; Mohal, N. Tetrahedron Lett. 2001, 42, 7663.

5. Hudlicky, T.; Gonzalez, D.; Gibson, D. T. Aldrichim. Acta 1999, 32, 35 and references cited therein.

6. All new compounds reported here were racemic and were characterized on the basis of their spectral data $\left({ }^{1} \mathrm{H}\right.$ and ${ }^{13} \mathrm{C}$ NMR, IR, Mass). Selected spectroscopic data ( $J$ in Hz). 15: $\delta_{\mathrm{H}}\left(300 \mathrm{MHz}, \mathrm{D}_{2} \mathrm{O}\right): 4.02(1 \mathrm{H}$, br s $), 3.95(1 \mathrm{H}$, ddd, $J 1.8,4.5,9.3), 3.46$ (1H, d 1/2 ABq, $J 6.9,10.8), 3.37$ $(1 \mathrm{H}, \mathrm{d} 1 / 2 \mathrm{ABq}, J 6,10.8), 3.29-2.97(4 \mathrm{H}$, series of $\mathrm{m})$, $1.77-1.62(3 \mathrm{H}, \mathrm{m}) ; \delta_{\mathrm{C}}\left(75 \mathrm{MHz}, \mathrm{D}_{2} \mathrm{O}\right): 72.4(\mathrm{CH}), 70.0$ $(\mathrm{CH}), 63.8\left(\mathrm{CH}_{2}\right), 46.0\left(\mathrm{CH}_{2}\right), 45.7\left(\mathrm{CH}_{2}\right), 42.2(\mathrm{CH}), 21.9$
$\left(\mathrm{CH}_{2}\right) .20: \delta_{\mathrm{H}}\left(300 \mathrm{MHz}, \mathrm{D}_{2} \mathrm{O}\right): 7.27(5 \mathrm{H}, \mathrm{m}$, br s like), $3.92(1 \mathrm{H}, \mathrm{m}), 3.8-3.76(1 \mathrm{H}, \mathrm{m}), 3.7-3.6(2 \mathrm{H}, \mathrm{m}, \mathrm{ABq}$ like $)$, $3.26(1 \mathrm{H}, \mathrm{d} 1 / 2 \mathrm{ABq}, J 6,11), 3.2(1 \mathrm{H}, \mathrm{d} 1 / 2 \mathrm{ABq}, J 7,11)$, 2.85 (1H, dd, $J$ 8.4, 13.2), 2.73 (1H, br dd, $J 13.5), 2.55$ (1H, br dd, $J 13.5), 2.3(1 \mathrm{H}, \mathrm{dd}, J 9,13.2), 2.0-1.9(1 \mathrm{H}$, $\mathrm{m}), 1.81-1.75(1 \mathrm{H}, \mathrm{m}), 1.4-1.32(1 \mathrm{H}, \mathrm{m}) ; \delta_{\mathrm{C}}(75 \mathrm{MHz}$, $\left.\mathrm{D}_{2} \mathrm{O}\right): 137.2(\mathrm{C}), 130.9(2 \mathrm{CH}), 129.4(2 \mathrm{CH}), 128.7(\mathrm{CH})$, $71.9(\mathrm{CH}), 71.4(\mathrm{CH}), 65.8\left(\mathrm{CH}_{2}\right), 62.7\left(\mathrm{CH}_{2}\right), 56.8\left(\mathrm{CH}_{2}\right)$, $55.3\left(\mathrm{CH}_{2}\right), 33.8(\mathrm{CH}), 33.3\left(\mathrm{CH}_{2}\right) .21: \delta_{\mathrm{H}}(300 \mathrm{MHz}$, $\left.\mathrm{D}_{2} \mathrm{O}\right): 4.08-4.02(2 \mathrm{H}, \mathrm{m}), 3.44(1 \mathrm{H}, \mathrm{d} 1 / 2 \mathrm{ABq}, J 5.4$, 11.4), 3.36-3.25 (3H, m), $3.10(1 \mathrm{H}, \mathrm{dd}, J 3,13.5), 2.81(1 \mathrm{H}$, dd, $J 11,13.5), 2.25-2.17(1 \mathrm{H}, \mathrm{m}), 1.87(1 \mathrm{H}$, ddd as dt, $J$ 5.1, 15.3), $1.52(1 \mathrm{H}$, ddd, $J 3.6,10.5,15.3) ; \delta_{\mathrm{C}}(75 \mathrm{MHz}$, $\left.\mathrm{D}_{2} \mathrm{O}\right): 70.8(\mathrm{CH}), 70.1(\mathrm{CH}), 64.7\left(\mathrm{CH}_{2}\right), 48.9\left(\mathrm{CH}_{2}\right), 47.4$ $\left(\mathrm{CH}_{2}\right), 33.5(\mathrm{CH}), 32.9\left(\mathrm{CH}_{2}\right) .27: \delta_{\mathrm{H}}\left(300 \mathrm{MHz}, \mathrm{D}_{2} \mathrm{O}\right)$ : 7.33-7.30 (5H, m), 4.06-4.0 (1H, m), 3.78-3.63 (3H, series of m), $3.53(1 \mathrm{H}, \mathrm{d} 1 / 2 \mathrm{ABq}, J 4.8,10.8), 3.37$ (1H, dd, $J$ 6.6, 11.4), 2.93-2.9 (1H, m, dd like), 2.82-2.77 (1H, br d, $J$ 13.8), 2.39-2.3 (2H, m), 2.0-1.96 (2H, m), 1.75-1.67 (1H, $\mathrm{m}) ; \delta_{\mathrm{C}}\left(75 \mathrm{MHz}, \mathrm{D}_{2} \mathrm{O}\right): 138.7(\mathrm{C}), 132.6(2 \mathrm{CH}), 131.1(2$ $\mathrm{CH}), 130.4(\mathrm{CH}), 69.9(\mathrm{CH}), 67.0(\mathrm{CH}), 65.5\left(\mathrm{CH}_{2}\right), 64.9$ $\left(\mathrm{CH}_{2}\right), 63.9\left(\mathrm{CH}_{2}\right), 55.7\left(\mathrm{CH}_{2}\right), 49.1(\mathrm{CH}), 45.5\left(\mathrm{CH}_{2}\right) .28$ : $\delta_{\mathrm{H}}\left(300 \mathrm{MHz}, \mathrm{D}_{2} \mathrm{O}\right): 4.21-4.18(1 \mathrm{H}, \mathrm{m}), 3.92-3.83(1 \mathrm{H}$, $\mathrm{m}), 3.69-3.62(1 \mathrm{H}, \mathrm{m}), 3.59-3.51(1 \mathrm{H}, \mathrm{m}), 3.36-3.25(2 \mathrm{H}$, $\mathrm{m}), 3.15-3.01(2 \mathrm{H}, \mathrm{m}), 2.21-2.11(1 \mathrm{H}, \mathrm{m}), 1.97-1.86(2 \mathrm{H}$, $\mathrm{m}) ; \delta_{\mathrm{C}}\left(75 \mathrm{MHz}, \mathrm{D}_{2} \mathrm{O}\right): 66.5(\mathrm{CH}), 63.3(\mathrm{CH}), 62.3\left(\mathrm{CH}_{2}\right)$, $52.0\left(\mathrm{CH}_{2}\right), 46.1\left(\mathrm{CH}_{2}\right), 45.3(\mathrm{CH}), 42.2\left(\mathrm{CH}_{2}\right)$.

7. Baxter, E. W.; Reitz, A. B. J. Org. Chem. 1994, 59, 3175. 8. (a) Butters, T. D.; van den Broeck, L. A. G. M.; Fleet, G. W. J.; Krulle, T. M.; Wormald, M. R.; Dwek, R. A.; Platt, F. M. Tetrahedron: Asymmetry 2000, 11, 113; (b) Lohray, B. B.; Bhushan, V.; Prasuna, G.; Jayamma, L.; Lakshmi, N. S.; Narayanareddy, K. Indian J. Chem. 1999, 38B, 1311 . 